Revista de la Escuela de Ciencias de la Educación. 2022, Año 18 1(17), 223 -242. Enero a junio. Di Piero, E. El mérito de ser autónomo: en torno a la igualdad de oportunidades y al tipo de estudiante esperado en un grupo de escuelas secundarias.

\title{
EL MÉRITO DE SER AUTÓNOMO: EN TORNO A LA IGUALDAD DE OPOTUNIDADES Y AL TIPO DE ESTUDIANTE ESPERADO EN UN GRUPO DE ESCUELAS SECUNDARIAS
}

\author{
THE MERIT OF BEING SELF-EMPLOYED: AROUND EQUAL OPOTUNITIES AND \\ THE KIND OF STUDENT EXPECTED IN A HIGH SCHOOL GROUP
}

Emilia Di Piero

Universidad Nacional de La Plata, Argentina

medipiero@gmail.com

Recibido: 23 de junio de 2020

Aprobado: 2 de noviembre de 2020

Publicado: 31 de diciembre de 2021

Cita sugerida: Di Piero, E. (2022). El mérito de ser autónomo: en torno a la igualdad de oportunidades y al tipo de estudiantes esperado en un grupo de escuelas secundarias. Revista de Escuela de Ciencias de la Educación. 1(17), 223-242.

\section{RESUMEN}

En este trabajo se indagan representaciones de docentes y actores clave en torno al tipo de estudiante esperado en un grupo prestigioso de escuelas secundarias dependientes de la Universidad Nacional de La Plata, en la ciudad capital de la Provincia de Buenos Aires, Argentina. Si bien el mérito como criterio de selección educativa presente en los exámenes de ingreso dejó de dirimir la admisión a las escuelas a partir del retorno de la democracia al país en los años '80, la ideología meritocrática persiste en las instituciones educativas en la actualidad. A partir del análisis de las representaciones mencionadas se analiza el tipo de estudiante esperado en estas escuelas: un estudiante "autónomo". Se concluye que, más allá de que se trata de secundarias que desde los años ' 80 cuentan con un sorteo público "abierto" como política de admisión, la noción de mérito perdura en el requisito de "autonomía" que actúa como premisa normalizadora y legitima desigualdades.

El enfoque metodológico implicó un estudio de caso múltiple. Entre los años 2012 y 2016 se realizaron observaciones, entrevistas en profundidad a trece actores "clave", sesenta y siete entrevistas semiestructuradas a docentes seleccionados aleatoriamente y análisis de documentos. 
Revista de la Escuela de Ciencias de la Educación. 2022, Año 18 1(17), 223 -242. Enero a junio. Di Piero, E. El mérito de ser autónomo: en torno a la igualdad de oportunidades y al tipo de estudiante esperado en un grupo de escuelas secundarias.

Palabras clave: Escuela secundaria - Autonomía - Igualdad de oportunidades - Mérito - Estudiantes.

\section{ABSTRACT}

In this work, representations of key teachers and actors about the type of student expected are investigated in a prestigious group of secondary schools dependent on the National University of La Plata, in the capital city of the Province of Buenos Aires, Argentina. Although merit as an educational selection criterion present in the entrance exams is no longer the mechanism for school admission after the return of democracy to the country in the 1980s, the meritocratic ideology persists in educational institutions today. From the analysis of the representations, analyze the type of student expected in these schools: an "autonomous" student. It is concluded that, beyond the fact that they are secondary schools that since the 1980s have had an "open" public draw as an admission policy, the notion of merit is redefined and persists through the demand for autonomy. This requirement acts as a standardizing premise.

The methodological approach involved a multiple case study. Between 2012 and 2016, were made observations, in-depth interviews with thirteen "key" actors, sixty-seven semi-structured interviews with randomly selected teachers and documentary analysis.

Keywords: Highschool - Autonomy - Equal opportunities - Merit - Highschool students.

\section{INTRODUCCIÓN}

La libertad absoluta lleva al aprovechamiento de esta desigualdad por parte de los aventajados, de los inteligentes frente a los más tontos, de los fuertes frente a los débiles, de los atrevidos frente a los tímidos

(Simmel, 2003. p.114).

La tensión entre las tendencias a la inclusión y a la selección social atraviesa los niveles medio y superior de los sistemas educativos en el mundo. La particularidad argentina ante ella fue la preferencia por la apertura general de los distintos niveles: heredando los resabios de cierto ethos igualitario sarmientino, con la recuperación democrática en los años ' 80 se intervino en los mecanismos de acceso a las escuelas secundarias y los exámenes de ingreso fueron -salvo excepciones ${ }^{1-}$ desterrados, siendo reemplazados en ciertos casos por el sorteo - específicamente mediante la Resolución No 2414/84-. El azar se erigía, así, como un criterio que apuntaba a la impugnación igualitarista a la meritocracia y recreaba la consigna del por entonces presidente Raúl Alfonsín en relación con la democratización de todos los ámbitos sociales: "con la democracia se come, se cura y se educa".

\footnotetext{
${ }^{1}$ En otros trabajos pueden encontrarse sistematizadas las secundarias preuniversitarias que sostienen el examen de ingreso eliminatorio en el marco del abanico heterogéneo que conforma este grupo de escuelas dependientes de universidades en Argentina (Di Piero, 2018 A).
} 
Revista de la Escuela de Ciencias de la Educación. 2022, Año 18 1(17), 223 -242. Enero a junio. Di Piero, E. El mérito de ser autónomo: en torno a la igualdad de oportunidades y al tipo de estudiante esperado en un grupo de escuelas secundarias.

De ese modo y a diferencia de otros países, en Argentina el acceso a los sucesivos niveles del sistema educativo se presenta como abierto y desregulado, siendo rechazados los exámenes de admisión por considerárselos modos antidemocráticos de selección social. En ese sentido, el tránsito de un nivel a otro aparece como "libre, abierto e irrestricto"2. Sin embargo, el hecho de que no exista una selección formal al momento de ingresar no significa que no se produzca selección alguna, sino que la misma se desarrolla de modos más implícitos y tácitos en comparación con otros países, dando lugar a aquello que algunos autores han denominado como "efecto colador" (Tiramonti y Ziegler, 2008), en referencia al desgranamiento que paulatinamente va sufriendo la matrícula una vez que los estudiantes se encuentran dentro de las instituciones.

Es decir que mientras otros modelos educativos sostienen la existencia de circuitos paralelos y diferenciados, el modelo argentino se asemeja a una pirámide en la cual muchos inician la escolaridad, pero se van cayendo del sistema sin que exista un examen de admisión que los excluya de manera formal en un comienzo, por lo cual ha sido caracterizado como un sistema de selección soft o sutil (Di Piero, 2015). En ese sentido, la noción de justicia vigente está en consonancia con la visión que Dubet (2012) conceptualiza como justicia por igualdad de oportunidades: el mérito relacionado con los resultados académicos continúa teniendo importancia en tanto factor de selección educativa pero ya no al momento del ingreso, sino en las trayectorias dentro de las instituciones. De ese modo, es en relación con el tránsito exitoso dentro de las escuelas que el grupo triunfante se legitima en un lugar de superioridad.

En ese sentido, en este trabajo ${ }^{3}$ se indagan representaciones de docentes y actores clave en torno al tipo de estudiante esperado en un grupo prestigioso de escuelas secundarias dependientes de la Universidad Nacional de La Plata, en la capital de la Provincia de Buenos Aires, Argentina. Si bien el mérito vinculado al desempeño académico como criterio de selección educativa encarnado en los exámenes de ingreso dejó de ser el criterio para la admisión escolar a partir del retorno de la democracia al país en los años 80 , la ideología meritocrática persiste en las instituciones educativas en la actualidad. A partir del análisis de las representaciones de docentes y actores clave se analiza el tipo de estudiante esperado en este grupo de escuelas.

El caso bajo estudio se sitúa en la capital de la provincia de Buenos Aires que - con un total de 654.324 habitantes ${ }^{4}$ - conforma la jurisdicción Gran La

\footnotetext{
2 Un caso que presenta rasgos similares en América Latina es el de Uruguay. Asimismo, cabe aclarar que una excepción parcial en el caso brasileño es constituido por la "Escola de Aplicação" de la Facultad de Educación de la Universidad de São Paulo a la cual se accede mediante sorteo pero de acuerdo con tres categorías: $1 / 3$ para hijos de profesores y funcionarios de la FEUSP, $1 / 3$ para hijos de profesores y funcionarios de la USP y $1 / 3$ para la comunidad en general. En el artículo 48 del reglamento actual se indica que las vacantes para la matrícula inicial en el nivel secundario serán reservadas automáticamente a los alumnos egresados del nivel primario de la EA (Di Piero y Mataluna, 2018).

${ }^{3}$ Este trabajo es parte de la tesis correspondiente al Doctorado en Ciencias Sociales de la Facultad Latinoamericana de Ciencias Sociales/ Sede académica Argentina defendida y aprobada en el año 2016.

${ }^{4}$ Disponible en: www.censo2010.indec.gov.ar/. Consultado el día: 09/03/2018.
} 
Revista de la Escuela de Ciencias de la Educación. 2022, Año 18 1(17), 223 -242. Enero a junio. Di Piero, E. El mérito de ser autónomo: en torno a la igualdad de oportunidades y al tipo de estudiante esperado en un grupo de escuelas secundarias.

Plata, la cual es parte del sistema educativo bonaerense, el más numeroso de Argentina. La ciudad presenta un perfil administrativo y universitario: es sede de la Universidad Nacional de La Plata, una de las Universidades más antiguas y prestigiosas del país, y de otras universidades tales como la Universidad Tecnológica Nacional, la Universidad Católica de La Plata y la Universidad del Este. Se trata de un espacio signado por una sociabilidad joven fuertemente vinculada al mundo educativo, cultural y artístico. La oferta educativa de nivel secundario en la ciudad está conformada por tres grupos: escuelas privadas, escuelas dependientes de la Provincia de Buenos Aires y escuelas universitarias.

De allí la relevancia que adquiere el caso abordado en este trabajo per se en tanto se abordan dos de los establecimientos secundarios de "pregrado" dependientes de la primera de las universidades mencionadas, establecimientos considerados como las mejores escuelas de la ciudad por buena parte del imaginario educativo platense.

La investigación se realizó en la ciudad de La Plata entre los años 2012 y 2016. Se trató de un estudio de caso múltiple centrado en las dinámicas que acontecen en escenarios particulares (Eisenhardt, 1989): en este caso, dos escuelas de pregrado dependientes de la Universidad Nacional de La Plata ubicadas en la ciudad homónima. Una de ellas adoptó completamente el mecanismo de sorteo como forma de ingreso desde mediados de los años ' 80 basándose en un discurso "democratizador". La otra adoptó ese mecanismo de admisión parcialmente en el momento de retorno de la democracia a Argentina, incorporándolo para el total de aspirantes sólo a partir del año 2015 en un contexto en que gravitó una narrativa en favor de la "inclusión" educativa. Entre los años 2012 y 2016 fueron realizadas observaciones, entrevistados en profundidad trece (13) actores "clave" para las instituciones; efectuadas sesenta y siete (67) entrevistas semiestructuradas a docentes seleccionados de modo aleatorio, revisadas las Actas Taquigráficas del momento en que se discutía la modificación del método de admisión en el Consejo Superior de la Universidad en los años ' 80 y recuperado un corpus compuesto por seis documentos institucionales (que incluyó datos sociodemográficos de las matrículas de las escuelas). En función del análisis que se desarrolla en este trabajo se recuperarán fragmentos de las 80 entrevistas y una selección de los documentos (más específicamente, de las páginas web institucionales). Los actores clave entrevistados en profundidad fueron miembros de los equipos directivos, secretarios/as académicos/as, jefes/as de los departamentos, secretarios/as de extensión de cada una de las escuelas y funcionarios/as de la universidad, mientras que se realizaron 67 entrevistas semiestructuradas a docentes considerando que se desempeñaran en disciplinas diversas: 32 entrevistas en la escuela 1 y 35 en la escuela 2.

\section{DESARROLLO}

\section{En torno a las escuelas}

El concepto de "fragmentación educativa" (Kessler, 2002; Tiramonti, 2004) apunta a analizar la fabricación de espacios organizados en torno a un 
Revista de la Escuela de Ciencias de la Educación. 2022, Año 18 1(17), 223 -242. Enero a junio. Di Piero, E. El mérito de ser autónomo: en torno a la igualdad de oportunidades y al tipo de estudiante esperado en un grupo de escuelas secundarias.

conjunto de valores, creencias, expectativas en relación con la escolaridad, capitales (materiales, sociales y culturales), estilos de vida, opciones religiosas que definen culturas institucionales y patrones de socialización que difieren entre sí. Es decir que no se trata solo de circuitos paralelos jerarquizables como apuntaba el concepto de segmentación (Braslavsky, 1985) sino de espacios múltiples que configuran un paisaje educativo caleidoscópico. Se trata de fragmentos inmersos en una dinámica de cierre social: cada uno de ellos se cierra sobre sí mismo, promoviendo una socialización "entre iguales". Tal como afirma Parkin (1964) retomando el enfoque weberiano, el concepto de cierre social refiere al "proceso mediante el cual las colectividades sociales buscan ampliar al máximo sus recompensas limitando el acceso a los recursos y oportunidades a un número restringido de candidatos" (p. 69). Ciertamente, las titulaciones, las calificaciones y los méritos académicos y profesionales constituyen, junto a la propiedad, los dos dispositivos principales de exclusión en las sociedades capitalistas modernas.

Las escuelas dependientes de universidades nacionales en Argentina rondan el medio centenar y presentan políticas de admisión, públicos y estilos institucionales diferentes, conformando un abanico amplio y heterogéneo.

Dentro de este grupo de escuelas es posible encontrar distintos subgrupos: un subgrupo con características marcadamente elitistas y meritocráticas, ligado a la matriz original del nivel secundario, otro subgrupo que se ha dejado permear por los imperativos de democratización del nivel y un tercer subgrupo de escuelas preuniversitarias creadas en el siglo XXI y tras la sanción de la obligatoriedad del nivel secundario, específicamente con la finalidad de "incluir" (Di Piero, 2018).

Las escuelas de "pregrado" que se abordan en este trabajo son parte del segundo de los grupos mencionados en tanto se trata de instituciones que, si bien fueron creadas bajo la matriz selectiva del nivel secundario, se dejaron permear por la tendencia hacia la democratización del nivel.

Asimismo, constituyen parte de un mismo fragmento en los términos antes definidos: a diferencia de las escuelas provinciales y privadas, se rigen por el sistema preuniversitario. Como indica una de las entrevistadas, directora saliente de la Escuela 1:

por el otro lado, las articulaciones que nosotros les hacemos hacer a los alumnos con pasantías académicas en la facultad. Bueno, después todo el tema de una currícula flexible donde pueden elegir materias y talleres, esa posibilidad de elección, el colegio preuniversitario con esas faltas que rigen por materia... son, a lo mejor, pequeños detalles que si una escuela dependiente de la provincia pudiera implementar sería fascinante... pequeños detalles que van, entre

comillas, entrenando a los pibes para eso... porque las otras escuelas siguen siendo muy paternalistas (Nadia, directora saliente, Escuela 1).

En síntesis, el sistema preuniversitario que diferencia a estas escuelas de aquellas privadas y de las dependientes de la Provincia de Buenos Aires implica 
Revista de la Escuela de Ciencias de la Educación. 2022, Año 18 1(17), 223 -242. Enero a junio. Di Piero, E. El mérito de ser autónomo: en torno a la igualdad de oportunidades y al tipo de estudiante esperado en un grupo de escuelas secundarias.

que las asistencias son computadas por horas y no por días de clase, la existencia de materias optativas en las cuales se mezclan estudiantes provenientes de distintos cursos habilitando una sociabilidad ampliada, la posibilidad de acceder a los establecimientos sin regulaciones estrictas de entrada o salida y de realizar pasantías en materias de la universidad. A continuación, se presenta en detalle cada una de las instituciones.

\section{La Escuela 1: "No era cualquier señorita"}

La Escuela 1 es uno de los tres establecimientos secundarios de pregrado de la UNLP situados en la ciudad de La Plata. Fue creada en 1907, dos años después de la nacionalización de la Universidad provincial, ante la demanda de una educación preuniversitaria femenina frente a la existencia del Colegio Nacional destinado únicamente a varones. De ese modo, sus orígenes resultan controvertidos puesto que, si bien se proponía atender a una elite, se dirigía a un subgrupo oprimido (teniendo en cuenta la variable género) dentro de sectores socioeconómicamente selectos: las mujeres. Como indicaban sus dos secretarias académicas, ambas egresadas de la institución, "no era cualquier señorita":

S: sí, no obstante, ese origen también era bastante interesante porque educar a las mujeres no era lo más corriente... y menos para que estudiaran carreras de grado.

R: claro, pero no era cualquier señorita.

S: era un pequeño grupo de señoritas.

R: y sí en ese momento... era contradictorio en su origen... que la mujer pueda entrar en la universidad pero que al mismo tiempo selecciona sólo a algunas (Rosana y Silvia, secretarias académicas, Escuela 1).

Son 844 los estudiantes que componen la matrícula de la escuela y 150 personas las que conforman su planta docente (el número asciende a 200 si se conciben como "docentes" también a los preceptores y ayudantes de los Departamentos).

Desde el año 1986 el ingreso a la escuela es directo, es decir, sin examen. Así, cuando la cantidad de inscriptas/os excede las vacantes previamente establecidas (hecho que va in crescendo año a año), se realiza un sorteo público entre las/os aspirantes: es el azar quien decide. Ingresan entonces 150 estudiantes distribuidos en 5 cursos de 30 estudiantes cada uno, si bien la cantidad precisa de vacantes a sortear depende del número de aspirantes provenientes de la Escuela primaria de la UNLP, quienes cuentan con prioridad para el ingreso en forma directa a cualquiera de las instituciones medias.

De este modo, el relato oficial de la institución describe a la implementación del sorteo como la bisagra que conllevó la heterogeneización de la matrícula ${ }^{5}$. A la nueva realidad que plantea el ingreso directo se añaden las metas propias de un nivel cuya obligatoriedad queda planteada en la Ley de

${ }^{5}$ Disponible en: http://www.escuela1.unlp.edu.ar/institucional Consultado el día: 04/03/2013. 
Revista de la Escuela de Ciencias de la Educación. 2022, Año 18 1(17), 223 -242. Enero a junio. Di Piero, E. El mérito de ser autónomo: en torno a la igualdad de oportunidades y al tipo de estudiante esperado en un grupo de escuelas secundarias.

Educación Nacional $\mathrm{n}^{\circ}$. 26206 del año 2006 y es sostenida por el estatuto vigente en la UNLP desde el año 2008.

\section{La Escuela 2}

Al igual que la otra institución, también la Escuela 2 se ubica en el casco céntrico de la ciudad. En este caso se trata de un bachillerato con especialidad artística que se propone articular conocimientos de las ciencias humanas y naturales con saberes relacionados con los lenguajes artísticos de la música y de las artes visuales.

La Escuela 2 data de una fundación más reciente. Sus comienzos se remontan al año 1949 con la creación del Ciclo Básico dentro del área de Extensión, que incorporaba estudiantes de escuelas primarias en los últimos tres años: las/os alumnas/os pasaban primero por el Ciclo Básico, luego por la Escuela de Dibujo y después por la Escuela Superior de Bellas Artes. En 1956 esta configuración se regularizó y fue creada la Escuela 2, que celebró en 2016 sus 60 años.

El primer plan de estudios fue aprobado con tres orientaciones en 1959: Dibujo Técnico, Dibujo Artístico y Música. Ante la preocupación por la salida laboral que la Escuela de Dibujo brindaba con su profesorado "secundario" se creó, entre 1960 y 1961, el Magisterio dentro del Bachillerato. Dicho título habilitó, hasta el año 2014, el ejercicio de la docencia en el nivel primario. Como indica el Plan de Estudios del año 2012, la organización curricular se estructura en dos campos: la Formación General y la Formación Específica.

Con respecto a la admisión, el Ciclo Básico de Formación Estética para quienes fueran sorteadas/os consistía en dos años de preparación: el mismo se cursaba tres veces a la semana desde las 18 horas en el caso de Discursos Musicales y dos veces en el caso de Discursos Visuales, en paralelo a la educación primaria. Hasta el año 2015, quienes no habían ingresado mediante sorteo podían rendir la "Evaluación de competencias de los lenguajes artísticos" para el ingreso a la Escuela en $1^{\circ}$ y $4^{\circ}$ año de la escuela secundaria. Dichas evaluaciones para ingresar en $1^{\circ}$ y $4^{\circ}$ año procuraban completar las vacantes que se generaban a partir de las deserciones producidas en el ciclo básico de formación estética ( $1^{\circ}$ año) y durante el trascurso de la ESB ( $4^{\circ}$ año). Los encuentros evaluatorios se extendían durante tres semanas y a partir de ello se establecía un orden de mérito. De este modo, hasta el ciclo lectivo 2015 la evaluación convivía con el sorteo para la admisión: a partir de entonces la admisión a la escuela es exclusivamente mediante el sorteo para el Ciclo Básico de Formación Estética.

\section{Una propuesta escolar con fuerte anclaje en la autonomía: procesos de individualización homogeneizantes}

Lejos de haber sido abandonada, la pretensión de homogeneización de la población escolar presente en el normalismo propio del momento de conformación del sistema educativo argentino persiste en muchas instituciones en la actualidad. En el caso del grupo de escuelas de pregrado de la UNLP, la promoción de una socialización con fuerte hincapié en la autonomía de los sujetos 
Revista de la Escuela de Ciencias de la Educación. 2022, Año 18 1(17), 223 -242. Enero a junio. Di Piero, E. El mérito de ser autónomo: en torno a la igualdad de oportunidades y al tipo de estudiante esperado en un grupo de escuelas secundarias.

aparece como el modo en que se tramita la tendencia homogeneizadora. Aunque resulte paradójico, el proceso de homogeneización del estudiantado en estas escuelas se realiza a partir de propiciar y promover la individualización.

Los gráficos que se presentan a continuación exhiben, agrupadas en porcentajes, las respuestas de las/os docentes cuando se les solicitó que definieran los rasgos característicos de un buen estudiante de la escuela. En el caso de la Escuela 1:

Gráfico 1. Escuela 1. Aspectos que definen a un "buen estudiante"

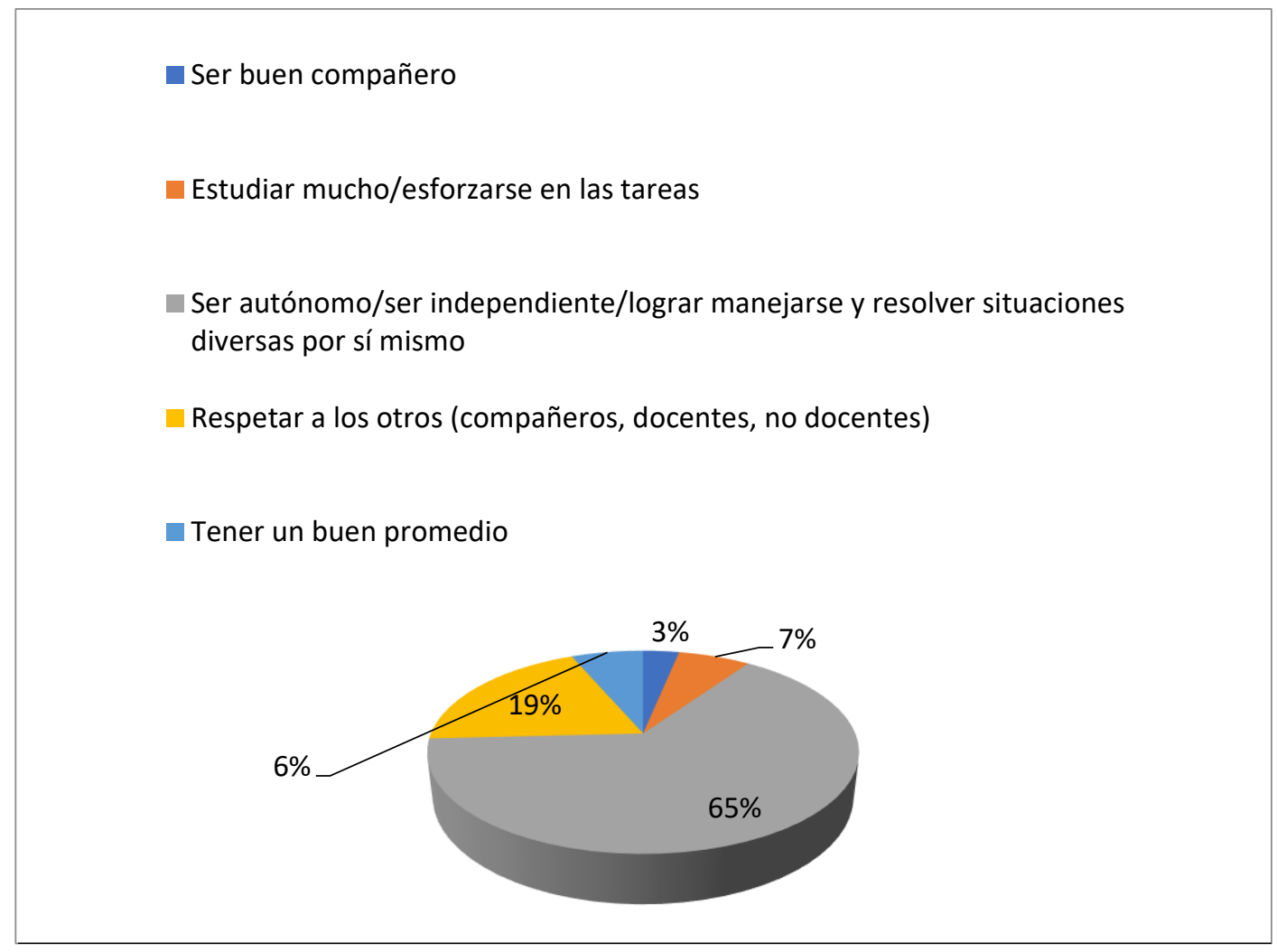

Fuente: elaboración propia

En cuanto a la Escuela artística:

Gráfico 2. Escuela 2. Aspectos que definen a un "buen estudiante" 
Revista de la Escuela de Ciencias de la Educación. 2022, Año 18 1(17), 223 -242. Enero a junio. Di Piero, E. El mérito de ser autónomo: en torno a la igualdad de oportunidades y al tipo de estudiante esperado en un grupo de escuelas secundarias.

- Ser buen compañero

- Ser autónomo/ser independiente/lograr manejarse y resolver situaciones diversas por sí mismo

- Tener capacidades artísticas/talento para el arte

Encajar en la propuesta que la escuela hace en general

Otro

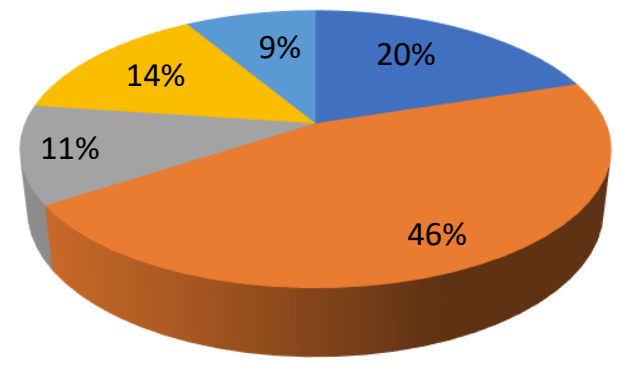

Fuente: elaboración propia

En la pauta de las entrevistas semiestructuradas se les pedía a los/as docentes que jerarquizaran sus respuestas, aunque optaran por respuestas múltiples. En los dos gráficos anteriores se presenta la opción que resultaba elegida en primera instancia. Como muestran los gráficos, un "buen estudiante" en estas escuelas es definido primordialmente por su grado de autonomía: así lo indica el $65 \%$ de los docentes consultados en la Escuela 1 y el $46 \%$ de los docentes en la Escuela 2. Esta opción es seguida por la opción de "respetar a los otros" en el caso de la Escuela 1 y de "ser buen compañero" en el caso de la Escuela 2, con más de 20 puntos porcentuales de diferencia entre los docentes que señalan la primera o la segunda opción en ambos casos. Entretanto, la cuestión del mérito vinculada al "buen promedio" figura con un porcentaje ínfimo.

En ese sentido, las representaciones de los docentes guardan afinidad con la propuesta oficial que rige en estas escuelas. Tal como figura en la página oficial de la Escuela 1:

[la Escuela] trabaja para que, a través de la circulación, transmisión y construcción del conocimiento, sus estudiantes desarrollen un pensamiento crítico, se eduquen en los valores de la cooperación y la solidaridad, en el respeto y la responsabilidad, que sean personas libres y autónomas, que sean participativos y ejerzan actitudes democráticas, que se comprometan con la realidad social, y que la transformen. 
Revista de la Escuela de Ciencias de la Educación. 2022, Año 18 1(17), 223 -242. Enero a junio. Di Piero, E. El mérito de ser autónomo: en torno a la igualdad de oportunidades y al tipo de estudiante esperado en un grupo de escuelas secundarias.

En efecto, uno de los ejes vertebradores de la propuesta es el empeño por inculcar ciertas pautas de socialización en el alumnado que permitan formar "estudiantes autónomos". Según indicaba Mónica, una de las dos vicedirectoras de la escuela artística:

Tiene que tener autonomía y poder adecuar el nivel de respuesta a la expectativa que le propone en general el colegio y cada profesor en particular. Y poder detectar qué es lo que importante en cada campo disciplinar para tener una respuesta ajustada a eso (...) Sigue estando la idea del bolillero en el que se te juega la vida si sabés o no sabés. Entonces, más allá de que nosotros podamos hacer un montón de estrategias, el final es que el alumno tiene que saber el programa. Entonces eso es lo que nos genera muchísima contradicción, porque además nosotros adherimos a que los chicos tienen que salir sabiendo, el tema es el camino que vamos tomando, ¿no? Y entonces ahí el alumno, hay un punto en el que está solo. (...) Un alumno de la universidad lo que tiene que lograr es esa autonomía en cada una de las disciplinas o en cada uno de los campos en donde se tiene que mover produciendo, siendo evaluado, siendo calificado, y aprobado o desaprobado... es una construcción llegar a entender todas estas lógicas. Y desde eso que sería lo fundamental hasta, bueno, el horario en donde es mejor ir a sacar fotocopias. Entonces ahí hay un montón de cosas, de aprendizajes subsidiarios, que hacen también al todo y que tienen que ver con dominar la escena. Y que hacen que vos, qué sé yo, después vayas a la facultad a inscribirte, entres a ese mundo que no entendés, pero que lo vas a entender rápidamente. Porque ya te volviste un estratega conociendo las reglas del juego. (...) Entonces empezar a entender eso para poder ir ganando lugares en ese campo. Ganando lugares antes era ser el mejor alumno, entrar a la facultad en las mejores condiciones. Hoy los pibes tal vez juegan más a poder sobrevivir (Mónica, vicedirectora, escuela 2).

De ese modo, Mónica se refería a que el/la estudiante de esta escuela debe tener capacidad de "sobrevivir" adaptándose a las diferentes situaciones que la institución y las/os docentes le van planteando. Como ella afirma, deben ser capaces de convertirse en "estrategas conociendo las reglas de juego" y de "dominar la escena y los aprendizajes subsidiarios".

También la secretaria de extensión de la Escuela 2 indicaba en ese sentido:

Esta escuela lo que ofrece es mucha posibilidad de elección para el alumno en su recorrido curricular, y esa capacidad hace que haya más de cuarenta modelos de analítico al finalizar cada promoción (Nina, Secretaria de Extensión, escuela 2).

Es decir que, recuperando lo señalado por los docentes y actores clave entrevistados, se entiende por autonomía la disposición de cada estudiante a gestionar su libertad de elección y darse a sí mismo las normas necesarias para transitar exitosamente la experiencia escolar, sin necesidad de que se lo esté supervisando de manera externa y permanente por parte de los adultos de la institución. 
Revista de la Escuela de Ciencias de la Educación. 2022, Año 18 1(17), 223 -242. Enero a junio. Di Piero, E. El mérito de ser autónomo: en torno a la igualdad de oportunidades y al tipo de estudiante esperado en un grupo de escuelas secundarias.

En sintonía con ello, en La autoridad, Richard Sennet problematiza la noción de autonomía en relación con la autoridad: refiere a este concepto como la posibilidad de tener autoridad sobre uno mismo. Señala que la persona autónoma es la que se domina a sí misma y por ello infunde respeto: la autonomía es, a su entender, la heredera del individualismo del siglo XIX. La persona autónoma puede decir a los otros lo que deben hacer porque la estructura de su personalidad hace que no busque la aprobación o supervisión de los demás 6 .

Por su parte, Lahire (2004) propone el concepto de "repertorios de hábitos" para discutir con la noción de habitus elaborada por Bourdieu, entendida como sistema de disposiciones duraderas y transferibles, estructuras estructuradas predispuestas a funcionar como estructuras estructurantes, es decir, como principios generadores y organizadores de prácticas y de representaciones (Bourdieu, 2010, p.86). Al entender de Lahire, distintas situaciones sociales se constituyen en activadores de los compendios de las experiencias incorporadas, de ciertas "disposiciones". Sin embargo, aclara que puede suceder que las fuerzas actuantes en los distintos contextos exijan cosas que no podemos darles, por no encontrarlas en los "stocks" o repertorios de esquemas de acción acumulados. Según cuáles sean los contextos que atravesamos, deberán reprimirse, inhibirse o activarse algunas competencias, maneras de decir y hacer que acarreamos.

Leyendo en esta clave el tipo de estudiante esperado en las escuelas de pregrado, la autonomía se exige como parte de sus stocks de disposiciones y opera, a su vez, como premisa normalizadora en relación con el tipo de estudiante esperado. La homogeneización, en el caso específico de estas instituciones, se produce a partir de un proceso de individualización de las/os estudiantes en el que gana peso el margen para la propia elección y en el que el mérito queda asociado a lograr un elevado grado de autonomía y no al promedio o rendimiento académico.

En palabras de una de las vicedirectoras entrevistada en la escuela artística:

El tema de la elección es algo recurrente. Nosotros tenemos algunos ejes que atraviesan todo el plan de estudios. Uno de ellos es la elección... la elección digamos, de alguna manera, de la mano de lo que significa la libertad...la producción básicamente en lo artístico, ¿no? La producción artística. Lo esperable seria que en todas las disciplinas se pueda organizar la propuesta académica desde la idea de la producción: la producción personal, la producción de cada uno. Y el otro eje es el desarrollo de competencias comunicativas. Esos serían como los pilares de la cuestión (...) Lo que sí tiene es un alto grado de optatividad todo el plan de estudios. Entonces, por ejemplo, en séptimo año tiene un paquete de diez horas, que los pibes lo eligen. Es decir, esas diez horas vos podés elegir cursarlas en ciencias exactas experimentales, podés elegir cursarlas en lengua, podés elegir cursarlas en ciencias sociales, o en el espacio de arte (Mónica, vicedirectora, escuela 2).

${ }^{6}$ Ciertamente, entre otras cuestiones, el autor muestra cómo se modifica el tipo de control ejercido sobre los empleados por parte de los jefes en la actualidad: desaparece la imagen del capataz duro y brutal y llega, en su lugar, la voz blanda del supervisor "orientado hacia las relaciones humanas" y el empleado se ve, sin embargo, motivado. 
Revista de la Escuela de Ciencias de la Educación. 2022, Año 18 1(17), 223 -242. Enero a junio. Di Piero, E. El mérito de ser autónomo: en torno a la igualdad de oportunidades y al tipo de estudiante esperado en un grupo de escuelas secundarias.

De ese modo, la vicedirectora hacía hincapié en la importancia de la libertad tanto en el momento de la elección de la escuela como durante las trayectorias en la misma.

Sin embargo, al entender la autonomía como una "disposición" o premisa con la cual las/os estudiantes deben contar, las instituciones de "pregrado" dejan a los/as estudiantes "libres" para "fracasar" en la resolución de cómo autoadministrar su libertad. Como señala Lahire: "los estudiantes de la universidad, y particularmente de las facultades menos controladas, se diferencian entre sí desde el punto de vista del grado de autodisciplina, del selfgovernment y de ascetismo que saben demostrar en función de su socialización familiar y escolar anteriores" (2004: 98). En ese sentido, el requisito de autonomía está en concordancia con el tipo de "promoción desregulada" al que refería Ziegler (2011).

En la otra cara de la moneda, quienes no consiguen adaptarse al tipo ideal de estudiante esperado desde el proyecto de las escuelas no son considerados autónomos. En consonancia con ello, un docente de Historia de 37 años de la Escuela 2, señalaba con respecto a los estudiantes que "no encajan". El que no logra la autonomía porque no encajó en la propuesta que la escuela les hace, se termina yendo. Esta escuela implica un compromiso grande por parte de los alumnos (Docente $n^{\circ} 12$, escuela 2 ).

Es decir que se espera que el "compromiso" de parte de los estudiantes se sitúe en "lograr la autonomía" necesaria para encajar en la propuesta escolar. En ese sentido, no se interpela a las/os estudiantes como sujetos heterogéneos sino como sujetos autónomos en un sentido monolítico. Es decir que no se consideran las diferencias que traen en sus biografías educativas, las distancias entre la propuesta escolar y las trayectorias educativas familiares o las distintas edades de quienes transitan la escolarización (que en el caso de la Escuela artística van desde los 12 hasta los 19 años). De este modo, la categoría moderna de "alumno" como un sujeto único, indiferenciado y monolítico reaparece entre enunciadoras/es que predican sobre ellos desde una pedagogía que, anclada en la modernidad, no distingue particularidades. En síntesis, si bien se promueve la libertad de elección, se trata de procesos de individualización homogeneizantes.

\section{El mérito de ser autónoma/o: meritocracia, autonomía e igualdad de oportunidades}

Antón Pirulero, cada cual que atienda a su juego, y el que no lo atienda, pagará una prenda

(Canción popular infantil).

Una de las funciones de la escolarización en las sociedades modernas occidentales apunta a legitimar la asignación de posiciones sociales que se presuponen equitativamente abiertas a la "carrera de los talentos". Hobsbawm (2003) conceptualiza como "carrera abierta al talento" a las nuevas formas de 
Revista de la Escuela de Ciencias de la Educación. 2022, Año 18 1(17), 223 -242. Enero a junio. Di Piero, E. El mérito de ser autónomo: en torno a la igualdad de oportunidades y al tipo de estudiante esperado en un grupo de escuelas secundarias.

distinción aparecidas tras las revoluciones burguesas del siglo XVIII contra los privilegios aristocráticos basados en la herencia.

Como señala la investigadora brasileña Livia Barbosa (2003), las tensiones entre igualdad y meritocracia son constitutivas de la ética del desempeño en las sociedades modernas. La ideología meritocrática se conforma como el principal criterio de jerarquización social en tanto postula que las posiciones de los individuos de una sociedad deben ser consecuencia del mérito de cada uno. Siguiendo a la autora, la meritocracia puede ser interpretada desde dos dimensiones: una negativa y otra positiva. La primera remite al rechazo a los privilegios hereditarios y en ese sentido encarna la lucha de las burguesías modernas frente al orden feudal. Entretanto, la dimensión afirmativa apunta a que la meritocracia se constituya como criterio básico de organización social vinculada a los talentos individuales.

Por su parte, Van Zanten (2008) refiere al "mito meritocrático" como uno de los mecanismos de cierre en la educación. De este modo, la meritocracia constituye un punto de apoyo principal en sistemas democráticos donde las desigualdades ligadas al sostenimiento de posiciones profesionales y sociales mejor remuneradas y más prestigiosas que otras se legitiman si se producen en el marco de una igualdad de oportunidades para obtenerlas y el valor de los individuos es sancionado por una institución social neutra como, se supone, sería la escuela.

En el caso de Argentina no se institucionalizaron, mayoritariamente, mecanismos meritocráticos para el acceso a bienes escasos y deseados, como sí sucede en la mayor parte de los países del mundo. En el ámbito específicamente educativo, el mérito ligado al rendimiento académico dejó de ser el criterio legítimo de sustento de las políticas de admisión a la mayoría de las escuelas secundarias y universidades a partir de la recuperación democrática en 1983. Las escuelas seleccionadas, si bien se rigen bajo la autonomía universitaria, se hicieron eco de las transformaciones que imprimía el clima de época y modificaron sus políticas de admisión desde el examen eliminatorio hacia el sorteo público. En ese sentido, el sorteo para el ingreso a estas escuelas es afín a una matriz nacional de educación ligada a un ideario igualitarista. Sin embargo, la contracara de ese igualitarismo formal es el carácter desregulado y desigual que presentan las trayectorias educativas reales.

Como muestra Young (1961) en su narración crítica de un mundo distópico (situado en 2033, es decir tres cuartas partes de siglo más tarde desde la visión del autor, pero a menos de quince años desde nuestro punto de vista actual), en el sistema "meritocrático" de educación todos se deben dar la misma oportunidad de acceder a trabajos concomitantes con sus habilidades. Sin embargo, la meritocracia nunca prometió igualdad, solo que las desigualdades se distribuirían más equitativamente, creando una suerte de "aristocracia de talento". Más allá de la inexistencia de exámenes de admisión en el grupo de secundarias universitarias, el mérito en tanto criterio de justicia sigue vigente, aunque redefinido: no se asienta en el desempeño académico, sino que se recrea a partir de la exigencia de autonomía a quienes ingresan. En ese sentido, al consultarle a una docente de Química de la Escuela 1 por los motivos que generan el 
Revista de la Escuela de Ciencias de la Educación. 2022, Año 18 1(17), 223 -242. Enero a junio. Di Piero, E. El mérito de ser autónomo: en torno a la igualdad de oportunidades y al tipo de estudiante esperado en un grupo de escuelas secundarias.

desgranamiento en la escuela, indicaba que se trata de estudiantes que "no encajan" en el sistema, por el requerimiento de autonomía que implican las escuelas preuniversitarias en comparación con las escuelas dependientes de la Provincia de Buenos Aires (que conforman la mayoría de escuelas secundarias de la ciudad):

[los estudiantes que abandonan estas escuelas] seguramente en las escuelas de provincia van a andar bien. Acá hay más materias, se enseña a pensar, a ser autónomo, por ejemplo, en la asistencia a las materias: ellos tienen que saber solos cuántas faltas les quedan. Esa autonomía unos la saben aprovechar, otros no, por eso hay desgranamiento

(Docente $\mathrm{N}^{\circ}$ 17, Escuela 1).

Es decir que, por una parte, aquellos estudiantes que no se correspondan con el tipo de estudiante esperado o, en términos de la docente, "no sepan aprovechar la autonomía", abandonarán la institución e irán en la búsqueda de una escuela que resulte más acorde a sus "disposiciones".

Por otro lado, la confianza de las escuelas preuniversitarias en la autorregulación de los estudiantes guarda relación con el sector sociocultural del que mayoritariamente provienen quienes ingresan: se amplía la libertad porque se da por sentado que actuarán como se espera que lo hagan. Como se ha mostrado en otros trabajos, se trata de estudiantes mayoritariamente provenientes de familias con trayectorias universitarias (Di Piero, 2020).

En su obra La estructura del discurso pedagógico. Clases, códigos y control Basil Bernstein (1993) clasifica a las pedagogías en dos tipos: visibles e invisibles. Las primeras ponen énfasis en la performance del niño, en su producto externo: la calificación se produce según los grados en que se ajusten a los criterios explícitamente regulados por el transmisor. En cuanto a las segundas, se caracterizan por una menor preocupación por la adaptación del adquiriente a una norma externa común: se ocupan en mayor medida de la adquisición, en tanto parten de que cada adquiriente es único e incomparable. En ese sentido, es menos probable en el caso de hijos de familias más desaventajadas que no ponen a disposición del niño las competencias requeridas por las pedagogías invisibles, que éste se autorregule en la escuela de acuerdo con los requisitos de la práctica pedagógica invisible, y es fácil que interprete de forma errónea tanto la práctica como su contexto pedagógico (Bernstein, 1993, p.93).

También Bourdieu señalaba, en cuanto a la relación entre el tipo de jerarquías y la clase social que, mientras más autoritaria es la relación pedagógica, más favorable es para los hijos de las clases dominadas, mientras que una relación democrática, igualitaria, es favorable a los privilegiados, porque saben cómo dominar una situación de laissez- faire (2010, p.146). Asimismo, Bourdieu y Darbel (2007) refieren a la autonomía en relación con el sector sociocultural de pertenencia al analizar el público asistente a los museos de arte en Europa. En ese caso, indican que la ausencia de toda indicación o guía que facilite la visita presupone un público culto. La existencia de flechas, tablas, guías, conferencistas y recepcionistas daría el derecho de ignorar y de estar presente como ignorante, o el derecho de los ignorantes de estar presentes, contribuyendo 
Revista de la Escuela de Ciencias de la Educación. 2022, Año 18 1(17), 223 -242. Enero a junio. Di Piero, E. El mérito de ser autónomo: en torno a la igualdad de oportunidades y al tipo de estudiante esperado en un grupo de escuelas secundarias.

a minimizar el sentimiento de inaccesibilidad de la obra y a que el espectador no se sienta indigno (2007, p.84).

Es decir que la apuesta a la autonomía y a la autovigilancia actúa como un mecanismo que legitima desigualdades socioeducativas en tanto selecciona a quienes saben o no saben "aprovechar la oportunidad". En ese sentido, estas posiciones están en consonancia con aquello que Walzer (1995) denomina "mito de la exclusión justa o justificada", aquella exclusión que se produce en una sociedad organizada exclusivamente sobre la igualdad de oportunidades y la ideología meritocrática. En este tipo de sociedad hipotética, las clases se dividirían en quienes serían capaces de aprovechar las oportunidades ofrecidas y quiénes serían incapaces de hacerlo.

Por su parte, Dubet presenta dos modelos de justicia social: el modelo de "igualdad de oportunidades" y el de "igualdad de posiciones". El primero refiere a la concepción que ofrece a todos la posibilidad de ocupar las mejores posiciones en función de un principio meritocrático. A diferencia del segundo, no busca reducir la desigualdad en las posiciones sociales sino anular las discriminaciones que perturbarían la competencia: no se discute la brecha entre las posiciones de los obreros y las de los ejecutivos, sino que los hijos de los obreros tengan tantas oportunidades de convertirse en ejecutivos como los hijos de estos últimos. Como indica el autor, en esta concepción de la justicia social se ofrece a todos la oportunidad de disputar las mejores posiciones, ocupación que se realizaría según un criterio meritocrático. Las desigualdades serían aceptadas como justas sólo en tanto las posiciones estarían en principio abiertas a todos y resultarían de una competencia entre individuos a los que se presume iguales en sus puntos de partida. En la metáfora del "juego de la silla" que propone Dubet (2012, p.57), se discute menos sobre la cantidad de sillas disponibles que sobre las maneras de ocuparlas y sobre la equidad y el criterio del árbitro: cada uno espera ser más rápido que los demás en el momento de saltar. Es decir que se apunta a disminuir las discriminaciones en el acceso a las posiciones y a que todos tengan formalmente las mismas oportunidades para dar el salto, pero no se cuestiona el orden de las posiciones, los criterios, ni las jerarquías resultantes.

En el caso de las escuelas abordadas, al sentido de lo justo subyace mayoritariamente la representación de la igualdad de oportunidades garantizada por un sorteo que habilitaría la supervivencia de quienes muestren el mérito de ser autónomos/as una vez dentro de la institución, sosteniendo, al mismo tiempo, que el sistema es abierto. De este modo, en este grupo de secundarias universitarias, la idea de "merecer" cobra valor ligada a la posibilidad de desenvolverse autónomamente, recreando a la meritocracia como ideología legitimadora de las desigualdades, aunque sin atarla al desempeño académico y favoreciendo un proceso de "acumulación de ventajas" (Saraví, 2015, p.37) según el cual quienes partan de disposiciones más cercanas a las que estas escuelas exigen serán, a su vez, quienes mejor consigan sostenerse.

La igualdad de oportunidades es, muchas veces, presentada como una ideología progresista que valora la capacidad en el trabajo y la idea de progreso personal a fuerza de talento y autonomía: se trata de la visión afirmativa de la meritocracia, en los términos ya citados de Barbosa (2003). En varias ocasiones 
Revista de la Escuela de Ciencias de la Educación. 2022, Año 18 1(17), 223 -242. Enero a junio. Di Piero, E. El mérito de ser autónomo: en torno a la igualdad de oportunidades y al tipo de estudiante esperado en un grupo de escuelas secundarias.

los entrevistados mencionan que los estudiantes deben "encajar" en la propuesta escolar o bien, en caso de que no "encajen", buscar otras instituciones en las que transitar su escolarización (se reitera la opción por las escuelas provinciales). Este señalamiento insistente podría estar mostrando que distintas escuelas promueven distintos tipos de estudiantes: en estas instituciones universitarias se pondera, en particular, la autonomía como rasgo esperado y "el mérito" en tanto criterio de asignación de posiciones continúa vigente, aunque se reformula: ya no refiere a quien obtiene mejores notas $y$, así, consigue ingresar mediante examen eliminatorio, sino que apunta a un tipo de estudiante que logra ser autónomo/a. Al mismo tiempo, se asume que en otras instituciones el tipo de estudiante esperado será otro, lo cual ratifica que se trata de un sistema educativo fragmentado.

Es decir que, para el caso de este grupo de escuelas secundarias, subyace mayoritariamente la representación de una igualdad de oportunidades garantizada por el sorteo, sosteniendo, así, que el sistema es abierto y alimentando la expectativa del ascenso social mediante la educación. En palabras de una funcionaria de la Universidad entrevistada:

Bueno, el sorteo dio así, el azar hizo que entraran dos chicos del Mercadito a la escuela 1. (...) Nosotros tenemos un chico del mercadito egresado de la escuela

1. Y eso es una medalla que guarda el proyecto XX [proyecto de extensión]

(Liliana, funcionaria de la UNLP).

"El mercadito" es un barrio de los alrededores de la ciudad de La Plata habitado por sectores populares. De ese modo, en este relato la ilusión del ascenso social es legitimada por la figura del becario o boursier al que refiriera Bourdieu: aquel que, a diferencia del heredero o héritier, no porta un capital cultural de origen acorde al esperado por la escuela y se abre paso pagando el costo de un proceso de resocialización. La figura del "pobre meritorio" (Gluz, 2006) transforma la excepción en regla y alimenta la idea de justicia dada por la igualdad de oportunidades, en tanto la desigualdad resultaría de las diferencias en el mérito y el esfuerzo individuales: si él pudo triunfar, entonces todos podían (y así lo garantiza el sorteo), pero no se esforzaron lo suficiente y esa exclusión es justa o legítima (Walzer, 1995). Como afirma Dubet (2015), hay un componente darwiniano en la igualdad meritocrática de oportunidades: vencedores y vencidos no se deben más que a sí mismos.

De ese modo, una vez abiertas las puertas, la legitimación de las desigualdades se produce a través de mecanismos implícitos dando lugar a una "selección sutil o soft" (Di Piero, 2015) que habilita trayectorias desreguladas en las que triunfan los más aptos o, en términos de los entrevistados, quienes "aprovechan la oportunidad" que la autonomía les brindaría. Retomando a una docente entrevistada: "El filtro se produce solo, naturalmente" (Docente $n^{\circ} 25$, Escuela Artística).

En ese sentido cabe retomar la noción de "capacidades" de Amartya Sen (1996), que busca ir más allá de un principio de libertad negativa basado en la no interferencia -tal como representa una política de admisión abierta-, 
Revista de la Escuela de Ciencias de la Educación. 2022, Año 18 1(17), 223 -242. Enero a junio. Di Piero, E. El mérito de ser autónomo: en torno a la igualdad de oportunidades y al tipo de estudiante esperado en un grupo de escuelas secundarias.

apostando a la libertad positiva que significaría la "capacidad" real de concretar un derecho -en este caso la escolarización secundaria-. Una política educativa que busque promover la igualdad real más allá de la igualdad de oportunidades formal no se situaría únicamente en la distribución de los bienes "poniendo a disposición" las vacantes, sino en garantizar que quienes accedan cuenten efectivamente con los recursos necesarios para transitar su escolarización, tomando a la autonomía como un propósito a alcanzar y no como un punto de partida.

\section{CONCLUSIÓN}

En este trabajo se indagó el tipo de estudiante esperado en un grupo prestigioso de instituciones de nivel secundario. A continuación, se recuperan algunas dimensiones que emergen del análisis desarrollado: por una parte, la redefinición del mérito ligado a la autonomía y, por otra, el imaginario educativo con el que este tipo ideal de estudiante se relaciona.

Con respecto a la primera dimensión, el tipo ideal de estudiante autónomo/a funciona como premisa normalizadora y constituye un mecanismo implícito en la legitimación de desigualdades socioeducativas, más allá de la garantía del acceso abierto que encarna el sorteo público como política de admisión. La exigencia de un alto grado de autonomía a los estudiantes demanda la presencia de disposiciones relacionadas con la adaptación y flexibilidad en sus "stocks", es decir con conocer "aprendizajes subsidiarios" que se dan por sabidos y resultan fundamentales para sostener la escolarización en estas instituciones. En ese sentido, el requisito de la autonomía descansa en una ideología meritocrática en tanto se encuentra íntimamente relacionada con la "capacidad" de mostrar la propia adaptación invisibilizando el peso de los soportes colectivos y de las socializaciones previas en el proceso de escolarización. No se trata de que estas instituciones hayan abandonado, junto a los exámenes de admisión, al principio del mérito como asignador de posiciones de privilegio y a la ideología meritocrática como legitimadora de las posiciones alcanzadas, sino de una redefinición sobre aquello que se valora como meritorio: el mérito no radica en el desempeño académico o en tener el mejor promedio sino en la capacidad de "ser autónomo/a".

Finalmente, la posibilidad de que el sorteo dirima el ingreso a escuelas de prestigio en el imaginario educativo de la capital de la provincia de Buenos Aires es afín a un ideario igualitarista. Se trata de una sociedad que, como indicaba Guillermo O'Donnell (1997) en un ensayo clásico en el que compara la sociedad argentina con la brasileña, es atravesada por un horizontalismo impugnador de las jerarquías. Así, la "configuración cultural nacional" (Grimson, 2007) opera como una frontera entre lo que es posible decir y lo que no: qué métodos son legítimos a los fines de la selección y cuáles deberían ser desterrados. En un país 
Revista de la Escuela de Ciencias de la Educación. 2022, Año 18 1(17), 223 -242. Enero a junio. Di Piero, E. El mérito de ser autónomo: en torno a la igualdad de oportunidades y al tipo de estudiante esperado en un grupo de escuelas secundarias.

como Argentina que, atravesado por sucesivos procesos inmigratorios 7 permanece ligado a una narrativa que lo describe como "el país de las oportunidades" y en el cual la educación ha colaborado con la promoción social de los más meritorios, legitimar la ideología meritocrática es posible si y sólo si se ha garantizado la igualdad de oportunidades para la competencia. Siguiendo a Pousadela (2007):

en Argentina, a diferencia de Brasil, la igualdad de oportunidades reclama que alguien se haga cargo de las desigualdades iniciales y desactive sus efectos, aun cuando ello suponga que la institución en cuestión (...) deba internalizar los elevados costos del proceso de nivelación (p.107).

En ese sentido, los ganadores se legitiman en esa posición por haber superado un filtro que se describe como "natural" en tanto actúa durante la escolarización y no en el momento de la admisión.

Es decir que, si bien en Argentina el acceso al sistema educativo se presenta mayoritariamente con carácter abierto y los exámenes de admisión son rechazados por considerárselos antidemocráticos, el mérito en tanto criterio y la meritocracia en tanto ideología continúan vigentes al poner en valor las "capacidades" y grados de autonomía de los/as estudiantes. En las instituciones analizadas, en pos de dar lugar a la libertad, la autonomía y la elección se promueven trayectorias desreguladas y desiguales en tanto cada quien queda librado a sus propios recursos para permanecer.

\section{REFERENCIAS}

Barbosa, L. (2003). Igualdade e Meritocracia. A ética do desempeño nas sociedades modernas. Fundacao Getulio Vargas Editora.

Bernstein, B. (1993). [1990]. La estructura del discurso pedagógico. Clases, códigos y control. Vol. IV. Morata.

Bolívar, A. (2012). Justicia social y equidad escolar. Una revisión actual. Revista Internacional de Educación para la Justicia Social, 1(1), 1-30.

Boltanski, L. y Chiapello, E. (2002). El nuevo espíritu del capitalismo. Akal.

Bourdieu, P. y Darbel, A. (2007). O amor pela arte. Os museus de arte na Europa e seu público. Editora da Universidade de São Paulo.

Bourdieu, P. (2010). El sentido práctico. Siglo XXI Editores.

Braslavsky, C. (1985). La discriminación educativa en Argentina. FLACSO- Grupo Editor Latinoamericano.

Bröckling (2015). El self emprendedor. Sociología de una forma de subjetivación. Ediciones Universidad Alberto Hurtado.

Canelo, P. (2019). ¿Cambiamos? La batalla cultural por el sentido común de los argentinos. Siglo XXI.

\footnotetext{
${ }^{7}$ Como muestra Paula Canelo, el mérito como criterio de justicia remite a la experiencia de ascenso social de los inmigrantes europeos y a la expresión " $M$ 'hijo, el dotor" de los cimientos de la Argentina moderna (2019, p.42).
} 
Revista de la Escuela de Ciencias de la Educación. 2022, Año 18 1(17), 223 -242. Enero a junio. Di Piero, E. El mérito de ser autónomo: en torno a la igualdad de oportunidades y al tipo de estudiante esperado en un grupo de escuelas secundarias.

Castel, R.; Kessler, G.; Murard, N. y Merklen. D. (2013). Individuación, precariedad, inseguridad. ¿Desinstitucionalización del presente? Editorial Paidós.

Chaves, M.; Fuentes, S. y Vecino, L. (2017) Experiencias juveniles de desigualdad: fronteras y merecimientos en sectores populares, medios altos y altos. CLACSO.

Di Piero, E. (2015). ¿Mérito y azar? Nociones de justicia distributiva y selección soft: el caso de una escuela secundaria tradicional frente al mandato inclusor. Revista Propuesta Educativa, 1(43) año 24, 152-154.

Di Piero, E. y Mataluna, M. (2018). Educación secundaria en instituciones dependientes de universidades públicas: miradas docentes que consolidan su prestigio en Brasil y Argentina. Trabajo y Sociedad, No 30.

Di Piero, E. (2018). Procesos de jerarquización en secundarias universitarias en Argentina. Universitas UPS, Revista de Ciencias Sociales y Humanas de la Universidad Politécnica Salesiana del Ecuador (28), 61 - 78.

Di Piero, E. (2020). La escolarización en secundarias universitarias en Argentina: la elección escolar entre los techos de cristal y el cierre social. Revista ProPosições, 31.

Dubet, F. (2012). Repensar la justicia social. Contra el mito de la igualdad de oportunidades. Siglo XXI.

Eisenhardt, K.M. (1989). Building Theories from Case Study Research. Academy of Management Review 4(14).

Grimson, A. (comp.) (2007). Introducción en Pasiones Nacionales. Política y Cultura en Brasil y Argentina. Edhasa.

Hobsbawn, E. (2003). La era de la revolución, 1789-1848. Editorial Crítica.

Kessler, G. (2002). La experiencia escolar fragmentada. Estudiantes y docentes en la escuela media en Buenos Aires. UNESCO, I.I.P.E.

Lahire, B. (2004). El hombre plural. Los resortes de la acción. Ediciones Ballaterra.

Litichever, L. (2012). ¿Qué se regula hoy en las escuelas? Una mirada sobre las prescripciones de los reglamentos de convivencia, Revista Iberoamericana de Educación 1(59), 1-10.

Parkin, F. (1964). El cierre social como exclusión en Marxismo y teoría de clases. Una crítica burguesa. Espasa Calpe.

O 'Donnell, G. (1997). ¿Y a mí, qué mierda me importa? Notas sobre sociabilidad y política en Argentina y Brasil. En G. O' Donnell, Contrapuntos. Ensayos escogidos sobre autoritarismo y democratización. Paidós.

Rawls, J. (1993). Teoría de la Justicia. Fondo de Cultura Económica.

Rosanvallon, P. (2012). La sociedad de iguales. Manantial.

Saraví, G. (2015). Juventudes Fragmentadas: Socialización, Clase y Cultura en la Construcción de la Desigualdad. FLACSO.

Sen, A. (1996). Capacidades y bienestar en M. Nussbaum y A. Sen (comp). La calidad de vida. Fondo de Cultura Económica.

Sennet, R. (1982). La autoridad. Alianza.

Simmel, G. (2003). Cuestiones fundamentales de sociología. Gedisa editorial.

Stake, R. (1995). Investigación con estudios de caso. Ediciones Morata. 
Revista de la Escuela de Ciencias de la Educación. 2022, Año 18 1(17), 223 -242. Enero a junio. Di Piero, E. El mérito de ser autónomo: en torno a la igualdad de oportunidades y al tipo de estudiante esperado en un grupo de escuelas secundarias.

Svampa, M. (2000). Desde abajo. Las transformaciones de las identidades sociales. Biblos.

Tiramonti, G. (comp.) (2004). La trama de la desigualdad educativa. Mutaciones recientes en la escuela media. Manantial.

Young, M. (1961). The Rise of Meritocracy. 1870-2033. Penguin Books.

Van Zanten (2008). ¿El fin de la meritocracia? Cambios recientes en las relaciones de la escuela con el sistema económico, político y social en E. Tenti Fanfani, (comp.), Nuevos temas en la agenda de política educativa. IIPE- UNESCO, Siglo XXI.

Walzer, M. (1993). Las esferas de la justicia. Fondo de Cultura Económica.

Ziegler, S. (2011). Entre la desregulación y el tutelaje: ¿̇acia dónde van los cambios en los formatos escolares? En Tiramonti, G. (Dir.). Variaciones sobre la forma escolar. Límites y posibilidades de la escuela media. Homo Sapiens. 